PROCEEDINGS OF THE

AMERICAN MATHEMATICAL SOCIETY

Volume 128, Number 1, Pages 5-15

S 0002-9939(99)04915-1

Article electronically published on September 9, 1999

\title{
ON MODULES WHICH FORCE HOMOGENEOUS MAPS TO BE LINEAR
}

\author{
P. R. FUCHS
}

(Communicated by Ken Goodearl)

\begin{abstract}
Let $R$ be a ring with identity. We characterize in terms of the left ideal structure of $R$ when every homogeneous map between nonsingular $R$ modules is linear and answer some earlier questions of the author that remained open.
\end{abstract}

\section{INTRODUCTION}

For a ring $R$ with identity and $R$-modules $V, W$ let $\mathrm{M}_{R}(V, W):=\{f, V \rightarrow$ $W \mid f(r v)=r f(v)$ for all $v \in V, r \in R\}$ denote the group of all $R$-homogeneous maps between $V$ and $W$.

In [1], U. Albrecht and J. Hausen completely described all semiprime Goldie rings $R$ such that $\mathrm{M}_{R}(V, W)=\operatorname{Hom}_{R}(V, W)$ for all nonsingular $R$-modules $V, W$. One major goal of the present paper is to extend this characterization to arbitrary nonsingular rings with identity (Theorem 3.5).

If $V=W$, then $\mathrm{M}_{R}(V, V)$ has the additional structure of a near-ring w.r.t. the composition of maps. This near-ring, which we denote by $\mathrm{M}_{R}(V)$, has been the object of several investigations.

In [2], the authors started to explore the class $\mathscr{R}$ of all rings $R$ with identity such that $\mathrm{M}_{R}(V)=\operatorname{End}_{R}(V)$ for all $R$-modules $V$ and characterized all semiperfect rings in $\mathscr{R}$.

Our present investigations also relate to this paper. There, it remained an open problem whether every ring of $\mathscr{R}$ contains a direct product of full matrix rings of size at least 2 (Corollary II.9 in [2]). By giving an example of a ring in $\mathscr{R}$ with no nontrivial idempotents we show that this is not true in general. Thus, the class $\mathscr{R}$ seems to be much bigger than expected.

According to Albrecht and Hausen, an $R$-module $V$ is called a ray for a class $\mathscr{C}$ of unitary $R$-modules if $\mathrm{M}_{R}(V, W)=\operatorname{Hom}_{R}(V, W)$ for all $R$-modules $W$ in $\mathscr{C}$. Call $V$ a ray if $V$ is a ray for the class of all unitary $R$-modules.

The following observation indicates how this concept is related to the class $\mathscr{R}$.

Received by the editors June 25, 1997 and, in revised form, January 27, 1998.

1991 Mathematics Subject Classification. Primary 16D10; Secondary 16D50, 16E50, 16S90.

Key words and phrases. Nonsingular module, injective hull, regular ring, maximal ring of quotients.

(C)1999 American Mathematical Society 
Proposition 1.1. For a ring $R$ with identity, the following are equivalent:

1. $R \in \mathscr{R}$

2. Every $R$-module is a ray.

Proof. For $f \in \mathrm{M}_{R}(V, W) \backslash \operatorname{Hom}_{R}(V, W)$ define $g: V \times W \rightarrow V \times W$ by $g(v, w)=$ $(0, f(v))$. One checks that $g \in \mathrm{M}_{R}(V \times W)$ and if $f\left(v_{1}+v_{2}\right) \neq f\left(v_{1}\right)+f\left(v_{2}\right)$, then $g\left(v_{1}+v_{2}, 0\right) \neq g\left(v_{1}, 0\right)+g\left(v_{2}, 0\right)$, hence $R \notin \mathscr{R}$.

We now give a brief overview of the paper. Section 2 starts with some general information as to when homogeneous maps can be lifted to the injective hull. Then we completely characterize all regular self-injective rings in $\mathscr{R}$ (Theorem 2.8). All of these results will be used again in section 3 , where the characterization result mentioned above will be proved.

\section{Lifting homogeneous MAPS to THE INJECTIVE HULL}

Conventions: All of our rings have an identity and all modules are unitary. Let $\mathrm{E}(V)$ denote the injective hull and $\operatorname{Sing}(V)$ the singular submodule of a module $V$.

In the following we utilize the maximal (or Johnson) ring of quotients of a (left) nonsingular ring. To avoid a reproduction of definitions, we only briefly fix our notation and refer to the approach of $\S 3.3$ in [5] for further concepts and results.

Let $M$ be a nonsingular $R$-module and $\widehat{M}=\mathscr{S} / \sim$ such that $\mathscr{S}=\left\{(L, h) \mid L \unlhd_{l} R\right.$ is essential and $h: L \rightarrow M$ is an $R$-homomorphism $\}$ and the equivalence $\sim$ is defined by $\left(L_{1}, h_{1}\right) \sim\left(L_{2}, h_{2}\right)$ iff $\exists L \unlhd_{l} R, L$ essential, and $L \subseteq L_{1} \cap L_{2}: h_{1} / L=h_{2} / L$. $[L, h]$ shall denote the equivalence class of $(L, h) \in \mathscr{S}$. If $r[L, h]=[(L: r), r h]$, where $r h:(L: r) \rightarrow M,(r h)(x)=h(x r)$, then $\widehat{M}$ is an $R$-module isomorphic to $\mathrm{E}(M)$. By taking $M=R$ for a nonsingular ring $R$ one obtains the maximal ring of quotients $\widehat{R}$ of $R$.

Further, if $V, W$ are $R$-modules and $f \in \mathrm{M}_{R}(V, W)$, then $f$ is called extendable if there exists a homogeneous map $\hat{f} \in \mathrm{M}_{R}(\mathrm{E}(V), \mathrm{E}(W))$ such that $\hat{f} / V=f$. We start by developing some criteria which ensure that homogeneous maps between nonsingular $R$-modules are extendable.

Proposition 2.1. For nonsingular R-modules $V, W$ and $f \in \mathrm{M}_{R}(V, W)$, the following are equivalent:

1. $f$ is extendable;

2. $f$ is linear on every submodule $S$ of $V$ which is contained in a cyclic $R$ submodule of $\mathrm{E}(V)$.

Proof. $1 \Rightarrow 2$ : Let $\hat{f} \in \mathrm{M}_{R}(\mathrm{E}(V), \mathrm{E}(W))$ be an extension of $f$. If $S \subseteq R v \cap V$ for some $v \in \mathrm{E}(V)$ and $s_{1}=r_{1} v, s_{2}=r_{2} v$ are elements of $S$, then $f\left(s_{1}+s_{2}\right)=$ $f\left(\left(r_{1}+r_{2}\right) v\right)=\hat{f}\left(\left(r_{1}+r_{2}\right) v\right)=\left(r_{1}+r_{2}\right) \hat{f}(v)=\hat{f}\left(r_{1} v\right)+\hat{f}\left(r_{2} v\right)=f\left(s_{1}\right)+f\left(s_{2}\right)$.

$2 \Rightarrow 1$ : We define $\hat{f}: \mathrm{E}(V) \rightarrow \mathrm{E}(W)$ as follows: If $[L, h] \in \mathrm{E}(V)$, where $L$ is an essential left ideal of $R$ and $h: L \rightarrow V$ is an $R$-homomorphism, then $\hat{f}([L, h])=$ $[L, f \circ h]$. If $\hat{h}: \mathrm{E}(R) \rightarrow \mathrm{E}(V)$ denotes the extension of $h$ to $\mathrm{E}(L)=\mathrm{E}(R)$, we have $h(L) \subseteq \hat{h}(R)=R \hat{h}(1)$, hence $f / h(L)$ is linear by our assumption. It follows that $f \circ h: L \rightarrow W$ is linear, thus $[L, f \circ h] \in \mathrm{E}(W)$.

If $\left(L_{1}, h_{1}\right) \sim\left(L_{2}, h_{2}\right)$, then there exists an essential left ideal $L \subseteq L_{1} \cap L_{2}$ with $h_{1} / L=h_{2} / L$, hence $f \circ h_{1} / L=f \circ h_{2} / L$, i.e., $\left(L_{1}, f \circ h_{1}\right) \sim\left(L_{2}, f \circ h_{2}\right)$. This shows $\hat{f}$ is well-defined. 
Now let $r \in R$ and $[L, h] \in \mathrm{E}(V)$. Then $\hat{f}(r[L, h])=[(L: r), f \circ(r h)]$, and since $(f \circ(r h))(x)=f(h(x r))=(r(f \circ h))(x)$, for $x \in(L: r)$, we obtain $\hat{f}(r[L, h])=$ $[(L: r), r(f \circ h)]=r[L, f \circ h]=r \hat{f}([L, h])$, i.e., $\hat{f}$ is homogeneous. By Remark 3.3.4 in [5], one can identify $V$ with the submodule $\left\{\left[R, \rho_{x}\right] \mid x \in V\right\}$ of $\widehat{V}$, where $\rho_{x}: R \rightarrow$ $V, \rho_{x}(r)=r x$ for $r \in R$. Finally, we need to prove that $\hat{f}$ extends $f$. For $x \in V$, $\left(f \circ \rho_{x}\right)(r)=f(r x)=r f(x)=\rho_{f(x)}(r)$, hence $\hat{f}\left[R, \rho_{x}\right]=\left[R, f \circ \rho_{x}\right]=\left[R, \rho_{f(x)}\right]$, which shows $\hat{f} / V=f$.

We also need the following useful observation, which has been stated on several occasions, see for instance Proposition 3.1b in [1].

Proposition 2.2. Let $V, W$ be R-modules, $W$ nonsingular and $f \in \mathrm{M}_{R}(V, W)$. If $f / S$ is linear for some essential submodule $S$ of $V$, then $f$ is an $R$-homomorphism.

Proposition 2.3. For a nonsingular ring $R$, the following are equivalent:

1. Every homogeneous map between nonsingular R-modules is extendable;

2. Every essential left ideal of $R$ is a ray for the class of nonsingular $R$-modules;

3. Every $R$-submodule of $\widehat{R}$ is a ray for the class of nonsingular $R$-modules.

Proof. $1 \Rightarrow 2$ : Since every essential left ideal $L$ is contained in the cyclic submodule $R$ of $\mathrm{E}(L)=\mathrm{E}(R)=\widehat{R}$, this is immediate from Proposition 2.1.

$2 \Rightarrow 3$ : Suppose $0 \neq S$ is a submodule of $\widehat{R}$ and $f: S \rightarrow W$ a homogeneous map, where $W$ is nonsingular. Let $J$ be a left ideal of $R$ such that $R \cap S \oplus J$ is essential, and define $g: R \cap S \oplus J \rightarrow W$ by $g(x+y)=f(x)$. Since $g$ is homogeneous on the essential left ideal $R \cap S \oplus J, g$ is linear by our assumption, thus, $f / R \cap S$ is linear. Observing that $R \cap S$ is an essential $R$-submodule of $S$, since $R$ is essential in $\widehat{R}=\mathrm{E}(R)$, we conclude by Proposition 2.2 that $f$ is linear.

Since $3 \Rightarrow 2$ is trivial it remains to show that $2 \Rightarrow 1$ : Let $f: V \rightarrow W$ be a homogeneous map and define $\hat{f}: \mathrm{E}(V) \rightarrow \mathrm{E}(W)$ by $\hat{f}([L, h])=[L, f \circ h]$. Note that by our assumption $f \circ h$ is linear for every $R$-homomorphism $h: L \rightarrow V$. Like in Proposition 2.1 one can show that $\hat{f}$ extends $f$.

Our next results, which will also be needed again in section 3, demonstrate why we are interested in extending a homogeneous map $f: V \rightarrow W$ to a map $\hat{f}: \mathrm{E}(V) \rightarrow \mathrm{E}(W)$.

Proposition 2.4. Let $R$ be nonsingular and $V, W$ nonsingular injective $R$-modules. Then $\mathrm{M}_{R}(V, W)=\mathrm{M}_{\widehat{R}}(V, W)$.

Proof. Recall that $V, W$ are $\widehat{R}$-modules (Proposition 3.3.20 in [5]). The proof is now essentially the same as the proof that $\operatorname{Hom}_{R}(V, W)=\operatorname{Hom}_{\widehat{R}}(V, W)$ and shall be omitted. Note that one containment is clear, since $R$ is a subring of $\widehat{R}$.

From structure theory one knows that $\widehat{R}$ is a regular self-injective ring. We are now able to characterize all regular self-injective rings which have the property that $\mathrm{M}_{R}(V, W)=\operatorname{Hom}_{R}(V, W)$ for all nonsingular $R$-modules $V, W$. This is precisely the reason why Proposition 2.4 turns out to be useful. However, before we can state and prove this theorem, a couple of preliminary results are needed. The proof of our first observation is obvious.

Proposition 2.5. Let $I$ be a nonempty set, $V_{i}, W_{i} R$-modules, and $f_{i}: V_{i} \rightarrow$ $W_{i}$ a homogeneous map for $i \in I$. Then $\prod_{i \in I} f_{i}: \prod_{i \in I} V_{i} \rightarrow \prod_{i \in I} W_{i}$ with $\left(\prod f_{i}\right)\left(v_{i}\right)_{i \in I}=\left(f_{i}\left(v_{i}\right)\right)_{i \in I}$ is homogeneous. 
Proposition 2.6. If $V, W$ are $R$-modules for a nonsingular ring $R$ and $f: V \rightarrow W$ is homogeneous, then

1. $V / \operatorname{Sing}(V)$ is a nonsingular $R$-module;

2. $\bar{f}: V / \operatorname{Sing}(V) \rightarrow W / \operatorname{Sing}(W)$ with $\bar{f}(v / \operatorname{Sing}(V))=f(v) / \operatorname{Sing}(W)$ is homogeneous.

Proof. 1. is well-known and follows from the fact that $R$ is nonsingular.

2. Let $v_{1}, v_{2} \in V$ such that $v_{1}-v_{2} \in \operatorname{Sing}(V) . L=\operatorname{Ann}_{R}\left(v_{1}-v_{2}\right)$ is an essential left ideal and if $l \in L$, then $l v_{1}=l v_{2}$, hence $l f\left(v_{1}\right)=f\left(l v_{1}\right)=f\left(l v_{2}\right)=l f\left(v_{2}\right)$, which shows that $L\left(f\left(v_{1}\right)-f\left(v_{2}\right)\right)=0$. But then $\operatorname{Ann}_{R}\left(f\left(v_{1}\right)-f\left(v_{2}\right)\right)$ is essential and we obtain $f\left(v_{1}\right)+\operatorname{Sing}(W)=f\left(v_{2}\right)+\operatorname{Sing}(W)$. Thus $\bar{f}$ is well-defined and clearly $\bar{f}$ is also homogeneous.

In order to obtain our final preliminary result, we shall consult Chapter 10 in [4] for some structure theorems on regular self-injective rings.

Also, we need to recall a concept which has been introduced in [2]. A set $E=$ $\left\{e_{1}, \ldots, e_{n}\right\}$ of mutually orthogonal idempotents of a ring $R$ is called complete if $\sum_{i=1}^{n} e_{i}=1$. Define an equivalence relation $\sim$ on $E$ by $e_{i} \sim e_{j}$ if $R e_{i}$ and $R e_{j}$ are isomorphic as $R$-modules. Then the number $m(E)$ is defined by

$$
m(E)=\min \{|C| \mid C \text { is an equivalence class w.r.t. } \sim\} .
$$

Proposition 2.7. Let $R$ be a regular self-injective ring. Then $R$ can be decomposed as $R=A \times I$, where $A$ is abelian regular and I contains a complete set $E$ of mutually orthogonal idempotents such that $m(E) \geq 2$.

Proof. We shall apply the following observation, which follows immediately from Lemma II.6 in [2]. If $S$ is a ring such that $S=S_{1} \times S_{2}$ and $S_{1}, S_{2}$ contain complete sets $E_{1}=\left\{e_{1}, \ldots, e_{n}\right\}, E_{2}=\left\{f_{1}, \ldots, f_{m}\right\}$ of mutually orthogonal idempotents with $m\left(E_{1}\right) \geq 2$ and $m\left(E_{2}\right) \geq 2$, then $E=\left\{\left(e_{1}, 0\right), \ldots,\left(e_{n}, 0\right),\left(0, f_{1}\right), \ldots,\left(0, f_{m}\right)\right\}$ is a complete set of mutually orthogonal idempotents in $S_{1} \times S_{2}$ with $m(E) \geq 2$.

We now use some results of Chapter 10 in [4]. By Proposition 10.21, $R=R_{1} \times R_{2}$, where $R_{1}$ is a directly finite ring and $R_{2}$ is purely infinite.

Theorem 10.16 in [4] says that $R_{2} \cong R_{2} \times R_{2}$ as $R_{2}$-modules, hence $R_{2}=L_{1} \oplus L_{2}$ for some left ideals $L_{1}, L_{2}$ of $R_{2}$ which are both isomorphic to $R_{2}$ as $R_{2}$-modules. If $e+g=1$ for orthogonal idempotents $e \in L_{1}, g \in L_{2}$, then $R_{2} e=L_{1} \cong L_{2}=R_{2} g$, which shows that $m(\{e, g\})=2$.

According to Theorem 10.13 and Proposition 10.28 in [4], the directly finite component $R_{1}$ can be decomposed as $R_{1}=R_{11} \times R_{12}$, where $R_{11}$ contains a faithful abelian idempotent and $R_{12} \cong R_{12} e \times R_{12} e$ as $R_{12}$-modules for some idempotent $e \in R_{12}$. Now the same argument we have applied to $R_{2}$ shows that $R_{12}$ contains a complete set $E$ of mutually orthogonal idempotents with $m(E)=2$.

Since $R_{1}$ is directly finite, both $R_{11}$ and $R_{12}$ are directly finite, hence $R_{11}=$ $\prod_{n \in \mathbb{N}} \mathrm{M}_{n}\left(A_{n}\right)$ for some abelian regular rings $A_{n}$ by Theorem 10.24. Here $\mathrm{M}_{n}\left(A_{n}\right)$ shall denote the matrix ring of size $n$ over the ring $A_{n}$.

Putting our results together, we obtain that $R=A \times I$, where $I=\prod_{n \geq 2} \mathrm{M}_{n}\left(A_{n}\right) \times$ $R_{12} \times R_{2}$ and $A$ is the abelian regular ring $A_{1}$.

By the proof of Theorem II.8 in [2] and our previous arguments, $\prod_{n \geq 2} \mathrm{M}_{n}\left(A_{n}\right)$, $R_{12}$, and $R_{2}$ contain complete sets $E$ of mutually orthogonal idempotents with $m(E) \geq 2$, hence the remark at the beginning of this proof yields our result. 
We are now ready for

Theorem 2.8. For a regular self-injective ring $R$, the following are equivalent:

1. $R \in \mathscr{R}$;

2. $\mathrm{M}_{R}(V, W)=\operatorname{Hom}_{R}(V, W)$ for all $R$-modules $V, W$;

3. $\mathrm{M}_{R}(V, W)=\operatorname{Hom}_{R}(V, W)$ for all nonsingular $R$-modules $V, W$;

4. $R$ contains a complete set $E$ of mutually orthogonal idempotents with $m(E) \geq$ 2;

5. No homomorphic image of $R$ is a division ring.

Proof. $1 \Leftrightarrow 2$ is Proposition 1.1. Since $2 \Rightarrow 3$ is trivial, we proceed to show that $3 \Rightarrow 4$ : If $R$ does not contain a complete set $E$ of mutually orthogonal idempotents with $m(E) \geq 2$, then $R=A \times I$ such that $A \neq 0$ by Proposition 2.7. Let $P \neq A$ be a prime ideal of $A$ and define $f_{P}: A / P \times A / P \rightarrow A / P \times A / P$ by $f_{P}\left(a_{1} / P, a_{2} / P\right)=$ $\left(a_{1} / P, a_{2} / P\right)$, if $\left(a_{1} / P, a_{2} / P\right) \in(A / P \times\{0\}) \backslash\{(0,0)\}$, and $f_{P}\left(a_{1} / P, a_{2} / P\right)=(0,0)$, otherwise. Since $A$ is abelian regular, $A / P$ is a division ring for all prime ideals $P \neq A$ of $A$, hence the proof of Lemma II.10 in [2] (or a direct verification) shows that $f_{P} \in \mathrm{M}_{A / P}(A / P \times A / P)$, but not linear. Also, $A / P \times A / P$ is an $A$-module by $a\left(a_{1} / P, a_{2} / P\right)=\left(a a_{1} / P, a a_{2} / P\right)$ and since both $A / P$ and $A$ act in the same way on $A / P \times A / P, \mathrm{M}_{A}(A / P \times A / P)=\mathrm{M}_{A / P}(A / P \times A / P)$, i.e., $f_{P}$ is $A$-homogeneous.

Let $\mathscr{I}=\{P \unlhd A \mid P \neq A$ is prime $\}$ and $V_{P}=A / P \times A / P$ for $P \in \mathscr{I}$. By Proposition 2.5, $\prod f_{P}: \prod V_{P} \rightarrow \prod V_{P}$ is $A$-homogeneous.

It is well-known that every regular ring is semiprime, hence the intersection of all prime ideals of $A$ is zero. Consequently, if $\left(u_{P}\right),\left(v_{P}\right),\left(w_{P}\right)$ are elements of $\prod V_{P}$ such that $u_{P}=(1 / P, 0 / P), v_{P}=(0 / P, 1 / P), w_{P}=(1 / P, 1 / P)$ for $P \in$ $\mathscr{I}$, then $\operatorname{Ann}_{A}\left(u_{P}\right)=\operatorname{Ann}_{A}\left(v_{P}\right)=\operatorname{Ann}_{A}\left(w_{P}\right)=0$. Let $\overline{\left(x_{P}\right)}$ denote the image of an element $\left(x_{P}\right) \in \prod V_{P}$ under the canonical epimorphism $\pi: \prod V_{P} \rightarrow$ $\left(\prod V_{P}\right) / \operatorname{Sing}\left(\prod V_{P}\right)$. Since every regular ring is nonsingular, it follows from Proposition 2.6 that $W:=\left(\prod V_{P}\right) / \operatorname{Sing}\left(\prod V_{P}\right)$ is a nonsingular $A$-module and $g$ : $W \rightarrow W$ defined by $g\left(\overline{\left(x_{P}\right)}\right)=\overline{\left(\prod f_{P}\right)\left(x_{P}\right)}=\overline{\left(f_{P}\left(x_{P}\right)\right)}$ is $A$-homogeneous. Since $\operatorname{Ann}_{A}((1 / P, 0 / P))=0$, we have $\overline{\left(u_{P}\right)} \neq 0$ in $W$, hence

$$
\begin{aligned}
g\left(\overline{\left(u_{P}\right)}+\overline{\left(v_{P}\right)}\right)=g\left(\overline{\left(u_{P}+v_{P}\right)}\right)=\overline{\left(f_{P}(1 / P, 1 / P)\right)} \\
\quad=0 \neq \overline{((1 / P, 0 / P))}=g\left(\overline{\left(u_{P}\right)}\right)+g\left(\overline{\left(v_{P}\right)}\right),
\end{aligned}
$$

which shows that $g$ is $A$-homogeneous but not linear.

Since $R=A \times I, W=\left(\prod V_{P}\right) / \operatorname{Sing}\left(\prod V_{P}\right)$ is an $R$-module by $(a, i) w=a w$ for $(a, i) \in A \times I$ and $w \in W$. From the fact that $W$ is a nonsingular $A$-module, it is easy to see that this $R$-module is nonsingular. Moreover, $g$ is clearly $R$-homogeneous, hence we arrive at a contradiction to our assumption 3 .

Since $4 \Rightarrow 1$ by Theorem II.7 in [2], we conclude that the conditions $1-4$ are equivalent. The implication $1 \Rightarrow 5$ follows from Lemma II.10 in [2], thus it remains to show $5 \Rightarrow 4$.

From Proposition 2.7, we know that $R=A \times I$, where $A$ is abelian regular, and $I$ contains a complete set $E$ of mutually orthogonal idempotents with $m(E) \geq 2$. If $A \neq 0$, then we have a prime ideal $P \neq A$ in $A$, hence $R /(P \times I)=A / P$ is a division ring. Consequently, $A=0$ by assumption 5 , and condition 4 follows. This completes the proof of Theorem 2.8. 


\section{A CharaCterization OF Rings FOR WHiCH HOMOGENEOUS MAPS BETWEEN NONSINGULAR MODULES ARE LINEAR}

We start by developing an internal criterion on the left ideals of the ring $R$ which allows us to decide whether homogeneous maps between nonsingular $R$-modules are extendable to the injective hull or not.

Let $L$ be a left ideal of a nonsingular $\operatorname{ring} R$ and define

$$
\mathscr{L}(a, b, L)=\sum_{l \in L}(R l: a) \cap(R l: b)
$$

for $a, b \in L$, where $(R l: a),(R l: b)$ denote the noetherian quotients, i.e., $(R l: a)=$ $\{r \in R \mid r a \in R l\}$.

For the sake of brevity, we shall say that $R$ admits extensions if every homogeneous map $f: V \rightarrow W$ between nonsingular $R$-modules $V, W$ is extendable (section 2 ), i.e., there exists a homogeneous map $\hat{f}: \mathrm{E}(V) \rightarrow \mathrm{E}(W)$ such that $\hat{f} / V=f$.

Theorem 3.1. If $\mathscr{L}(a, b, L)$ is an essential left ideal of $R$ for every essential left ideal $L$ of $R$ and $a, b \in L$, then $R$ admits extensions.

Proof. Let $f: V \rightarrow W$ be a homogeneous map between nonsingular $R$-modules $V, W$. In order to show that $f$ is extendable we need to prove, according to Proposition 2.1, that $f$ is linear on every submodule $S$ of $V$ which is contained in a cyclic $R$-submodule $R v$ of $\mathrm{E}(V)$.

Since $V$ is essential in $\mathrm{E}(V), L:=(V: v)$ is an essential left ideal of $R$. If $v_{1}, v_{2} \in S$, then $v_{1}=a v, v_{2}=b v$ for some $a, b \in L$ since $S \subseteq R v \cap V$. Now let $w:=f(a v+b v)-f(a v)-f(b v)$ and $r \in \mathscr{L}(a, b, L)$. Then there exist a positive integer $n, r_{1}, \ldots, r_{n} \in R$, and $l_{1}, \ldots, l_{n} \in L$ such that $r=r_{1}+\cdots+r_{n}$ and $r_{i} a \in R l_{i}, r_{i} b \in R l_{i}$ for $i \in\{1, \ldots, n\}$. Fix $i \in\{1, \ldots, n\}$ and let $r_{i} a=s l_{i}, r_{i} b=t l_{i}$. Then $r_{i} w=r_{i}(f(a v+b v)-f(a v)-f(b v))=f\left(r_{i} a v+r_{i} b v\right)-f\left(r_{i} a v\right)-f\left(r_{i} b v\right)=$ $f\left(s l_{i} v+t l_{i} v\right)-f\left(s l_{i} v\right)-f\left(t l_{i} v\right)$. Since $l_{i} \in L, l_{i} v \in V$, hence $f\left(l_{i} v\right)$ is defined. But then the last expression equals $(s+t) f\left(l_{i} v\right)-s f\left(l_{i} v\right)-t f\left(l_{i} v\right)=0$ and we conclude that $r w=\left(r_{1}+\cdots+r_{n}\right) w=0$ for every $r \in \mathscr{L}(a, b, L)$.

Since $\mathscr{L}(a, b, L)$ is essential, $w \in \operatorname{Sing}(W)=0$, hence $f\left(v_{1}+v_{2}\right)=f\left(v_{1}\right)+f\left(v_{2}\right)$, i.e., $f$ is linear on $S$. By Proposition 2.1, $f$ is extendable.

Corollary 3.2. Every regular ring admits extensions.

Proof. Since $R$ is regular, $R$ is a nonsingular ring and every finitely generated left ideal is principal. Thus, if $L$ is an essential left ideal of $R$ and $a, b \in L$, then $R a+R b=R l$ for some $l \in L$, hence $R=(R l: a) \cap(R l: b) \subseteq \mathscr{L}(a, b, L)$.

A combination of this result with our main theorem, Theorem 3.5, will allow us to characterize all regular rings with the property that homogeneous maps between nonsingular modules are linear (Corollary 3.7).

If $L$ is an essential left ideal of a commutative ring $R$ and $a, b \in L$, then $l a$ and $l b$ are elements of $R l$ for every $l \in L$, hence $L \subseteq \mathscr{L}(a, b, L)$, which shows that $\mathscr{L}(a, b, L)$ is essential for all $a, b \in L$.

Corollary 3.3. Every commutative ring admits extensions.

However, our next result shows that there exist nonsingular rings which do not admit extensions. Recall that a ring $R$ is uniform ( $R$ has Goldie dimension 1$)$ if the intersection of two nonzero left ideals is always nonzero. 
Theorem 3.4. For an integral domain $R$, the following are equivalent:

1. $R$ admits extensions;

2. $R$ is uniform.

Proof. $1 \Rightarrow 2$ : Suppose $R$ is not uniform, say $R x \cap R y=0$ for some $x \neq 0, y \neq 0$. It is well-known that such a domain must have infinite Goldie dimension. Now let $L=R x \oplus R y$, and define $f: L \rightarrow L$ by $f(r x+s y)=0$ if $r \neq 0$ and $s \neq 0$, and $f(r x+s y)=r x+s y$, otherwise. One checks that $f$ is $R$-homogeneous but not linear, hence $R$ does not admit extensions by Proposition 2.3, part 3 .

$2 \Rightarrow 1$ : If $R$ is uniform, then $R x$ is an essential left ideal for all nonzero $x \in R$. Now let $L$ be a nonzero left ideal of $R$ and $a, b \in L$. By the previous remark, $(R l: a) \cap(R l: b)$ is essential for every $0 \neq l \in L$, hence $\mathscr{L}(a, b, L)$ is essential. Our claim now follows from Theorem 3.1.

We are heading towards a characterization of those rings for which homogeneous maps between nonsingular modules are linear. For this we need certain direct limits of modules which we are now going to describe.

As usual, take a nonsingular ring $R$ and let $L$ be a left ideal of $R$. We define a direct limit which corresponds to $L$. As an index set take $I=L \times L$ and associate to each $i=(a, b) \in I$ the $R$-module $V_{i}=R / \mathscr{L}(a, b, L)$.

Moreover, we have a preorder $\leq$ (i.e., a reflexive and transitive relation) on $I$ defined by $(a, b) \leq(c, d): \Leftrightarrow R(a, b) \subseteq R(c, d)$, where $R(a, b)$ and $R(c, d)$ denote the $R$-submodules of $R \times R$ generated by $(a, b),(c, d)$. Let $i=(a, b), j=(c, d)$ be elements in $I$ such that $i \leq j$. Then there exists $x \in R$ with $(a, b) \in(x c, x d)$ and we obtain a map $\varphi_{i}^{j}: V_{i} \rightarrow V_{j}$ defined by $\varphi_{i}^{j}(r / \mathscr{L}(a, b, L))=r x / \mathscr{L}(c, d, L)$. If we can show that $\varphi_{i}^{j}$ is well-defined, then clearly $\varphi_{i}^{j} \in \operatorname{Hom}_{R}\left(V_{i}, V_{j}\right)$.

In fact, if $r / \mathscr{L}(a, b, L)=r^{\prime} / \mathscr{L}(a, b, L)$, then $r-r^{\prime} \in \mathscr{L}(a, b, L)$, hence there exist $s_{1}, \ldots, s_{k} \in R, l_{1}, \ldots, l_{k} \in L$ such that $r-r^{\prime}=s_{1}+\cdots+s_{k}$ and $s_{i} a, s_{i} b \in R l_{i}$ for $i \in\{1, \ldots, k\}$. Since $(a, b)=(x c, x d), s_{i} x c, s_{i} x d \in R l_{i}$, hence $r x-r^{\prime} x=$ $s_{1} x+\cdots+s_{k} x \in \mathscr{L}(c, d, L)$.

Next we point out that $\varphi_{i}^{j}$ does not depend on the special choice of $x$. In fact, if $(a, b)=(x c, x d)==\left(x^{\prime} c, x^{\prime} d\right)$, then $\left(x-x^{\prime}\right) c=\left(x-x^{\prime}\right) d=0$, hence $x-x^{\prime} \in \operatorname{Ann}(c) \cap \operatorname{Ann}(d) \subseteq \mathscr{L}(c, d, L)$, i.e., $r x+\mathscr{L}(c, d, L)=r x^{\prime}+\mathscr{L}(c, d, L)$ for all $r \in R$.

From this we clearly obtain that $\varphi_{i}^{i}: V_{i} \rightarrow V_{i}$ is the identity map. Thus, in order to show $\left(V_{i} ; \varphi_{i}^{j}\right)_{i \in I}$ is a system, it remains to prove that $\varphi_{i}^{k}=\varphi_{j}^{k} \circ \varphi_{i}^{j}$, whenever $i \leq j \leq k$.

Let $i=(a, b) \leq j=(c, d) \leq k=(e, f)$, say $(a, b)=(x c, x d)$ and $(c, d)=$ $(y e, y f)$. Then $(a, b)=(x y e, x y f)$, hence $\varphi_{i}^{k}(r / \mathscr{L}(a, b, L))=r x y / \mathscr{L}(e, f, L)=$ $\varphi_{j}^{k}(r x / \mathscr{L}(c, d, L))=\varphi_{j}^{k}\left(\varphi_{i}^{j}(r / \mathscr{L}(a, b, L))\right)$.

Thus $\left(V_{i} ; \varphi_{i}^{j}\right)_{i \in I}$ is a system in the category of $R$-modules. Proposition 1.8.5 in [5] shows that the direct $\operatorname{limit}_{\lim _{L}}:=\lim _{\longrightarrow}\left(V_{i} ; \varphi_{i}^{j}\right)_{i \in I}$ always exists.

Moreover, if $\mu_{j}: V_{j} \rightarrow \bigoplus_{i \in I} V_{i}$ denotes the injection into the $j$-th component of $\bigoplus_{i \in I} V_{i}$, then

$$
\lim _{L} \cong \bigoplus_{i \in I} V_{i} / N
$$


where $N$ is the $R$-submodule $\sum_{i \leq j}\left(\mu_{j} \varphi_{i}^{j}-\mu_{i}\right)\left(V_{i}\right)$. Each injection $\mu_{j}$ induces an $R$ homomorphism $\overline{\mu_{j}}: V_{j} \rightarrow \lim _{L}$ for $j \in I$ in a natural way, namely $\overline{\mu_{j}}(v)=\mu_{j}(v) / N$. Moreover, one has that $\overline{\mu_{i}}=\overline{\mu_{j}} \circ \varphi_{i}^{j}$, whenever $i \leq j$.

We are now ready to state our main theorem.

Theorem 3.5. For a nonsingular ring $R$, the following are equivalent:

1. $M_{R}(V, W)=\operatorname{Hom}_{R}(V, W)$ for all nonsingular $R$-modules $V, W$;

2. (a) No homomorphic image of $\widehat{R}$ is a division ring,

(b) $\lim _{L}$ is a singular $R$-module for each left ideal $L$.

Proof. $1 \Rightarrow 2($ a): Let $V, W$ be nonsingular $\widehat{R}$-modules. Then $V, W$ are nonsingular $R$-modules since $R$ is an essential $R$-submodule of $\widehat{R}$. We have $\mathrm{M}_{\widehat{R}}(V, W)=$ $\mathrm{M}_{R}(V, W)=\operatorname{Hom}_{R}(V, W)=\operatorname{Hom}_{\widehat{R}}(V, W)$. The first equality follows as in Proposition 2.4, the second is the assumption, and the third is well-known. The result is now a consequence of Theorem 2.8 .

$1 \Rightarrow 2\left(\right.$ b): Suppose $\operatorname{Sing}\left(\lim _{L}\right) \neq \lim _{L}$ for some left ideal $L$. Define $f: L \times L \rightarrow$ $\lim _{L}$ by $f(c, d)=\bar{\mu}_{j}(1 / \mathscr{L}(c, d, L))$, for $j=(c, d) \in I=L \times L$.

Let $r \in R$ and $i=(r c, r d) \leq j$. Then

$$
\begin{aligned}
r f(c, d) & =r \bar{\mu}_{j}(1 / \mathscr{L}(c, d, L))=\bar{\mu}_{j}(r / \mathscr{L}(c, d, L)) \\
& =\bar{\mu}_{j} \circ \varphi_{i}^{j}(1 / \mathscr{L}(r c, r d, L))=\bar{\mu}_{i}(1 / \mathscr{L}(r c, r d, L))=f(r c, r d) .
\end{aligned}
$$

Thus $f$ is an $R$-homogeneous map and consequently $g: L \times L \rightarrow \lim _{L} / \operatorname{Sing}\left(\lim _{L}\right)$ defined by $g(c, d)=f(c, d) / \operatorname{Sing}\left(\lim _{L}\right)$ is $R$-homogeneous. Since $\lim _{L} \neq \operatorname{Sing}\left(\lim _{L}\right)$, there exists $j=(c, d) \in I$ with $\bar{\mu}_{j}(1 / \mathscr{L}(c, d, L)) / \operatorname{Sing}\left(\lim _{L}\right) \neq 0$ for otherwise the submodule $\operatorname{Sing}\left(\lim _{L}\right)$ would contain $\bar{\mu}_{j}\left(V_{j}\right)$ for all $j \in I$ and therefore $\lim _{L}$.

If $i=(c, 0)$, then $g(c, 0)=\bar{\mu}_{i}(1 / \mathscr{L}(c, 0, L)) / \operatorname{Sing}\left(\lim _{L}\right)$. However, since $c \in$ $L, \mathscr{L}(c, 0, L)=\sum_{l \in L}(R l: c) \cap(R l: 0)=R$, hence $g(c, 0)=0$ and similarly $g(0, d)=0$. However, $g(c, d) \neq 0$, thus $g(c, d) \neq g(c, 0)+g(0, d)$. This contradicts our assumption 1 , since $R$ is nonsingular and therefore $L \times L, \lim _{L} / \operatorname{Sing}\left(\lim _{L}\right)$ are nonsingular $R$-modules by Proposition 2.6.

$2 \Rightarrow 1$ : Let $V, W$ be nonsingular $R$-modules and $f: V \rightarrow W$ a homogeneous map. First we shall prove that $f$ is extendable. According to Proposition 2.1, it suffices to show that $f$ is linear on every submodule $S$ of $V$ which is contained in a cyclic $R$-submodule of $\mathrm{E}(V)$.

So suppose $f$ is not linear on $S \subseteq R v \cap V$ for some $v \in \mathrm{E}(V)$. Since $V$ is an essential submodule of $\mathrm{E}(V), L=(V: v)$ is an essential left ideal of $R$ and $S \subseteq L v$. We may now construct the left ideals $\mathscr{L}(a, b, L)$ for $L=(V: v)$ and the $R$-homomorphisms $\varphi_{i}^{j}$, where $i, j \in I=L \times L$, and as we have outlined before, $\lim _{L}$ exists.

Define $g_{i}: V_{i}=R / \mathscr{L}(a, b, L) \rightarrow \mathrm{E}(V)$ for $i=(a, b) \in I$ by $g_{i}(r / \mathscr{L}(a, b, L))=$ $r(f(a v+b v)-f(a v)-f(b v))$. We show $g_{i}$ is an $R$-homomorphism for all $i \in I$.

Indeed, if $r-r^{\prime} \in \mathscr{L}(a, b, L)$ and $w=f(a v+b v)-f(a v)-f(b v)$, then we proceed as in the proof of Theorem 3.1 to show that $r-r^{\prime} \in \operatorname{Ann}_{R}(w)$, hence $r w=r^{\prime} w$. Thus $g_{i}$ is well-defined and clearly it is also an $R$-homomorphism.

Now let $x \in R, j=(a, b) \in L \times L$ and $i=(x a, x b)$, hence $i \leq j$. Then $g_{j} \circ \varphi_{i}^{j}(r / \mathscr{L}(x a, x b, L))=g_{j}(r x / \mathscr{L}(a, b, L))=r x(f(a v+b v)-f(a v)-f(b v))=$ $r(f(x a v+x b v)-f(x a v)-f(x b v))=g_{i}(r / \mathscr{L}(x a, x b, L))$ for all $r / \mathscr{L}(x a, x b, L) \in$ $R / \mathscr{L}(x a, x b, L)$. This shows that $g_{i}=g_{j} \circ \varphi_{i}^{j}$ for all $i \leq j \in I$ and by the properties 
of a direct limit there exists a unique $R$-homomorphism $g: \lim _{L} \rightarrow \mathrm{E}(V)$ such that $g \circ \bar{\mu}_{i}=g_{i}$ for all $i \in I$.

We started with the assumption that $f$ is not linear on some submodule $S \subseteq L v$. So choose $i=\left(r_{1}, r_{2}\right) \in L \times L$ such that $w:=f\left(r_{1} v+r_{2} v\right)-f\left(r_{1} v\right)-f\left(r_{2} v\right) \neq 0$.

Then

$$
\begin{aligned}
g \circ \bar{\mu}_{i}\left(1 / \mathscr{L}\left(r_{1}, r_{2}, L\right)\right) & =g_{i}\left(1 / \mathscr{L}\left(r_{1}, r_{2}, L\right)\right) \\
& =f\left(r_{1} v+r_{2} v\right)-f\left(r_{1} v\right)-f\left(r_{2} v\right)=w .
\end{aligned}
$$

By our assumption 2, $\operatorname{Sing}\left(\lim _{L}\right)=\lim _{L}$, hence $A:=\operatorname{Ann}_{R}\left(\bar{\mu}_{i}\left(1 / \mathscr{L}\left(r_{1}, r_{2}, L\right)\right)\right)$ is essential, and for $r \in A$ we have

$$
r w=r\left(g \circ \bar{\mu}_{i}\right)\left(1 / \mathscr{L}\left(r_{1}, r_{2}, L\right)\right)=g\left(r \bar{\mu}_{i}\left(1 / \mathscr{L}\left(r_{1}, r_{2}, L\right)\right)\right)=g(0)=0 .
$$

This shows that $\operatorname{Ann}_{R}(w)$ is an essential left ideal and since $W$ is nonsingular, $w=0$. Thus $f\left(r_{1} v+r_{2} v\right)=f\left(r_{1} v\right)+f\left(r_{2} v\right)$, which contradicts our assumption.

We have now shown that every $R$-homogeneous map $f: V \rightarrow W$ between nonsingular $R$-modules is extendable to a map $\hat{f}: \mathrm{E}(V) \rightarrow \mathrm{E}(W)$ and by Proposition $2.4 \hat{f} \in \mathrm{M}_{\widehat{R}}(\mathrm{E}(V), \mathrm{E}(W))$. Our assumption 2 together with Theorem 2.8 yields $\hat{f} \in$ $\operatorname{Hom}_{\widehat{R}}(\mathrm{E}(V), \mathrm{E}(W))$, thus we may finally conclude that $\mathrm{M}_{R}(V, W)=\operatorname{Hom}_{R}(V, W)$ for all nonsingular $R$-modules $V, W$, which completes the proof of our theorem.

Remark 3.6. 1. The proof of $2 \Rightarrow 1$ in Theorem 3.5 shows that we can replace condition 2(b) by

$\mathbf{2} \mathbf{b}^{\prime}: \lim _{L}$ is a singular $R$-module for each essential left ideal $L$.

2. If $\mathscr{L}(a, b, L)$ is an essential left ideal for all $(a, b) \in I=L \times L$, then $V_{i}$ is a singular module for all $i \in I$, hence $\lim _{L}=\bigoplus_{i \in I} V_{i} / N$ is singular.

We now apply Theorem 3.5 to the class of regular rings. If $L$ is a left ideal of a regular ring $R$, then it follows from the proof of Corollary 3.2 that $\mathscr{L}(a, b, L)=R$ for all $a, b \in L$, hence $\lim _{L}=0$ by Remark 3.6. Since every regular ring is nonsingular, we obtain

Corollary 3.7. For a regular ring $R$, the following are equivalent:

1. $\mathrm{M}_{R}(V, W)=\operatorname{Hom}_{R}(V, W)$ for all nonsingular $R$-modules $V, W$;

2. No homomorphic image of $\widehat{R}$ is a division ring.

As we have noted before, U. Albrecht and J. Hausen characterized all semiprime Goldie rings $R$ such that $\mathrm{M}_{R}(V, W)=\operatorname{Hom}_{R}(V, W)$ for all nonsingular $R$-modules $V, W$ (Theorem 3.5 in [1]). Let us quickly discuss how this characterization is related to our Theorem 3.5. Every semiprime Goldie ring is nonsingular and $\widehat{R}$ coincides with the classical ring of quotients, hence $\widehat{R}$ is a direct product of matrix rings over division rings. Then our condition 2 (a) says that the size of all matrix rings in this product is greater or equal to 2. Every essential left ideal $L$ of $R$ contains a regular element $t$ and $R t$ is also essential. But then $(R t: a) \cap(R t: b)$ is essential for all $a, b \in L$, which in turn implies that $\mathscr{L}(a, b, L)$ is an essential left ideal for all $a, b \in L$. Thus $\lim _{L}$ is singular for every essential left ideal $L$ by Remark 3.6 and we see that the result of Albrecht and Hausen coincides with Theorem 3.5 for semiprime Goldie rings (if condition 2(b) is replaced by $2 \mathrm{~b}^{\prime}$ of Remark 3.6).

Our final result for this paper is related to the class $\mathscr{R}$, which has been introduced in [2]. There, it remained an open problem whether $\mathscr{R}$ contains rings without 
nontrivial idempotents. A combination of some of our previous results in this paper shows this is indeed the case.

Theorem 3.8. A simple (left) noetherian ring $R$ is contained in the class $\mathscr{R}$ if and only if $R$ is not an integral domain.

Proof. By Lemma II.10 in [2], no integral domain is contained in the class $\mathscr{R}$. So let $R$ be a simple (left) noetherian ring which is not an integral domain, and suppose there exist an $R$-module $V$ and a homogeneous map $f \in \mathrm{M}_{R}(V) \backslash \operatorname{Hom}_{R}(V)$, say $w=f\left(v_{1}+v_{2}\right)-f\left(v_{1}\right)-f\left(v_{2}\right) \neq 0$ for some $v_{1}, v_{2} \in V$.

Since $\operatorname{Ann}(R w)$ is an ideal of $R$ and $w \neq 0$, we must have that $\operatorname{Ann}(R w)=0$. Let $V_{r}=V, f_{r}=f$ for $r \in R$ and $V_{1}=\prod_{r \in R} V_{r}$. By Proposition 2.5, $g:=\prod_{r \in R} f_{r}$ : $V_{1} \rightarrow V_{1}$ is a homogeneous map, hence $g \in \mathrm{M}_{R}\left(V_{1}\right)$. If $x=\left(r v_{1}\right)_{r \in R}, y=\left(r v_{2}\right)_{r \in R}$, then $u:=g(x+y)-g(x)-g(y)=\left(f\left(r v_{1}+r v_{2}\right)-f\left(r v_{1}\right)-f\left(r v_{2}\right)\right)_{r \in R}=(r w)_{r \in R}$. From $\operatorname{Ann}(R w)=0$ it follows that $\operatorname{Ann}_{R}(u)=0$, hence $u \notin \operatorname{Sing}\left(V_{1}\right)$.

Define $\bar{g}: V_{1} / \operatorname{Sing}\left(V_{1}\right) \rightarrow V_{1} / \operatorname{Sing}\left(V_{1}\right)$ by $\bar{g}\left(v / \operatorname{Sing}\left(V_{1}\right)\right)=g(v) / \operatorname{Sing}\left(V_{1}\right)$. Since every simple ring is nonsingular, Proposition 2.6 shows that

$$
\bar{g} \in \mathrm{M}_{R}\left(V_{1} / \operatorname{Sing}\left(V_{1}\right)\right)
$$

and $V_{1} / \operatorname{Sing}\left(V_{1}\right)$ is nonsingular. If $\bar{x}=x / \operatorname{Sing}\left(V_{1}\right), \bar{y}=y / \operatorname{Sing}\left(V_{1}\right)$, then $\bar{g}(\bar{x}+\bar{y})-\bar{g}(\bar{x})-\bar{g}(\bar{y})=(g(x+y)-g(x)-g(y)) / \operatorname{Sing}\left(V_{1}\right)=u / \operatorname{Sing}\left(V_{1}\right)$. As we have noted above, $u \notin \operatorname{Sing}\left(V_{1}\right)$, hence $\bar{g}(\bar{x}+\bar{y}) \neq \bar{g}(\bar{x})+\bar{g}(\bar{y})$, which shows that $\bar{g} \in \mathrm{M}_{R}\left(V_{1} / \operatorname{Sing}\left(V_{1}\right)\right) \backslash \operatorname{Hom}_{R}\left(V_{1} / \operatorname{Sing}\left(V_{1}\right)\right)$.

We can now apply Theorem 3.5 or, since $R$ is a semiprime Goldie ring, Theorem 3.5 in the paper of Albrecht and Hausen (see the remarks after Corollary 3.7). The Johnson ring $\widehat{R}$ of quotients of a simple ring $R$ is also simple and since $R$ is not integral, $\widehat{R}$ must be a matrix ring of size greater or equal to 2 over a division ring. Thus every nonsingular $R$-module is a ray for the class of all nonsingular $R$-modules by Theorem 3.5 in [1], which contradicts our result that $\bar{g} \in \mathrm{M}_{R}\left(V_{1} / \operatorname{Sing}\left(V_{1}\right)\right) \backslash \operatorname{Hom}_{R}\left(V_{1} / \operatorname{Sing}\left(V_{1}\right)\right)$. We conclude that our original assumption $\mathrm{M}_{R}(V) \neq \operatorname{Hom}_{R}(V)$ was false, hence $R \in \mathscr{R}$.

Corollary 3.9. The class $\mathscr{R}$ contains rings without nontrivial idempotents.

Proof. The ring $R$ constructed by A. E. Zalesskii and O. M. Neroslavskii in [6] (consult also [3] for an exposition) is simple, noetherian, not integral, and without nontrivial idempotents. By Theorem 3.8, $R \in \mathscr{R}$.

In particular, this shows that $\mathscr{R}$ contains rings which do not possess a complete set $E$ of mutually orthogonal idempotents with $m(E) \geq 2$.

\section{ACKNOWLEDGMENT}

The author wants to thank the referee for his/her comments, which helped to shorten some of the proofs.

\section{REFERENCES}

1. U. Albrecht and J. Hausen, Non-singular modules and R-homogeneous maps, Proc. Amer. Math. Soc. 123 (1995), 2381-2389. MR 95j:16026

2. P. Fuchs, C. J. Maxson, and G. Pilz, On rings for which homogeneous maps are linear, Proc. Amer. Math. Soc. 112 (1991), 1-7. MR 91h:16054 
3. K. R. Goodearl, Simple noetherian rings, the Zalesskii-Neroslavskii examples, in ring theory, Lecture Notes in Mathematics (New York-Berlin) (D. Handelman and J. Lawrence, eds.), no. 734, Springer-Verlag, 1978, pp. 118-130. MR 81b:16009

4. — Von Neumann regular rings, Monographs and Studies in Mathematics, no. 4, Pitman, London, 1979. MR 80e:16011

5. L. H. Rowen, Ring theory, Vol. I, Pure and Applied Mathematics, vol. 127, Academic Press, San Diego, 1988. MR 89h:16001

6. A. E. Zalesskii and O. M. Neroslavskii, There exist simple noetherian rings with zero divisors but without idempotents, Comm. Algebra 5 (1977), 231-244. MR 55:12761

Department of Mathematics, Johannes Kepler University, A-4040 Linz, Austria

E-mail address: peter.fuchs@jk.uni-linz.ac.at 